\title{
OSCILLATIONS OF MULTILAYER VISCOELASTIC COMPOSITE TOROIDAL PIPES
}

\author{
Muhsin Kh. Teshaev ${ }^{1 *}$, Ismail I. Safarov ${ }^{2}$, Mirziyod Mirsaidov ${ }^{3}$ \\ ${ }^{1}$ Bukhara Engineering-Technological Institute; \\ e-mail: muhsin_5@mail.ru \\ ${ }^{2}$ Tashkent Chemical-Technological Institute, \\ ${ }^{3}$ Tashkent Institute of Engineers of Irrigation and Mechanization at Agriculture \\ *corresponding author
}

\begin{abstract}
This article discusses a method for calculating the dynamic characteristics of thin-walled viscoelastic composite pipes made by continuous winding under the influence of internal hydrodynamic pressures. The solution is based on an approximate energy method based on the instant theory of shells and the second-order Lagrange equation. Initial technological irregularities are taken into account. The pipe is considered a multilayer viscoelastic toroidal shell with variable reinforcement parameters, initial ovality and difference in thickness. The dynamics problem is formulated as the problem of natural oscillations of a compressed thin-walled structure. The basic equations are obtained in the form of a coupled system of ordinary integrodifferential equations with variable stiffness coefficients.
\end{abstract}

Keywords: pipeline, pressure, semi-moment less theory, parametric oscillation, freezing method.

\section{Introduction}

Pipelines occupy a special place in aviation, rocket and aerospace engineering. They are an integral part of the design of propulsion systems, ground launch complexes, power equipment. The total length of the hydraulic, air and fuel systems for designed production of a modern aircraft is several kilometers, their number is several thousand (Hambartsumyan 1974; Kubenko et al. 2003). The modern thin-walled pipeline of large diameter with internal pressure of more than 10 $\mathrm{MPa}$ is not an ordinary rod or beam, but a thin cylindrical shell with a deformable contour of the cross section. Therefore, to calculate such a pipeline should not use the traditional core theory, but the theory of thin shells, taking into account the interaction of the structure with the internal and external environment. The reliability of pipelines significantly determines the operability of flying vehicles in general. Therefore, the pipelines are subjected to increased requirements for reliability, resource and, at the same time, strict restrictions on weight and dimensions. These conflicting requirements are best analyzed by thin-walled multilayer composite structures. One of the most widespread and advanced methods of manufacturing multilayer pipes from fibrous composite materials is the method of continuous winding (Kubenko et al. 1992). Reinforced tape formed by a system of threads is impregnated with a polymer binder. Then it is fed to a rotating 
mandrel and laid along predetermined paths. For fiber laying, detachable rotating heads and programmed automatic machines are used. After polymerization of the binder and removal of the mandrel, a multilayer shell of a given shape is formed. The most technologically challenging are curvilinear pipes made of fibrous composite materials. In the process of manufacturing curved pipes by continuous winding of fibers or reinforced tape, an inhomogeneous layered and fibrous structure with variable angles and reinforcement coefficients is formed (Kovalchuk and Kruk 2000). Typical technological irregularities of the product are ovality and thickness variation of the cross section. At the same time, curved sections are the most stressed elements of pipelines. The main load is internal pressure. Tests of the film pipeline under pressure showed that failure occurs as a result of rupture of the shell in the zone of thinning of the wall of a curved section (Safarov et al. 2018a). Wall ruptures are one of the main causes of pipeline failures: An important advantage of pipes made of fibrous composite materials is the non-fragmentation nature of failure. The problem of nonlinear vibrations of thin cylindrical shells, taking into account the interaction of various bending forms, was discussed in Safarov et al. $2018 \mathrm{~b}$ and Safarov and Boltaev 2018. In Safarov et al. 2018c and Amabili et al. 2000, the features of the influence of a liquid aggregate (partial filling) on the processes of dynamic interaction of the forms of bearing shells were studied.

In this paper, we consider the problem of multimode vibrations of composite toroidal shells (orthotropic model) completely filled with liquid. The main attention is paid to the study of the interaction under resonance conditions of conjugated and non-conjugated bending forms of these shells under free vibrations of the entire shell-liquid system.

An alternative solution can be implemented using the finite element method. Then, an 8-node curvilinear isoperimetric finite element method can be used. The composite shell is considered as a degenerate three-dimensional anisotropic layered body.

\section{Problem statement and solution methods}

The dynamic state of thin-walled viscoelastic composite curved pipes manufactured by continuous winding under the influence of internal hydrodynamic pressures is considered.

To derive resolving equations describing the damped oscillations of curved (toroidal) composite pipes under the influence of hydrodynamic pressure, we use the semi-moment less theory of shells V.Z. Vlasov (1949). The limits of applicability of semi-moment less theories for solving the problems of statics and dynamics of thin-walled pipes were studied in Stasenko and Rakhmanova (1986) and Khaletskaya (1975). Moreover, it is believed that the curved pipe is quite long, slightly bent and thin-walled. Given the weak heterogeneity of the physic mechanical properties of individual layers, the semi-moment less theory has also been used to calculate shells of fibrous composites (Vasiliev 1988; Biderman 1980). We consider that wall deformations, as a packet of layers, obey the Kirchhoff - Love hypothesis. Taking into account the kinematic relations of the semi-moment less theory of thin shells and the symmetry condition, we present the following forms of motion:

$$
\begin{aligned}
& w(s, \theta, t)=\sum_{k=1}^{\infty}\left[w_{1 k}(s, t) \cos k \theta+w_{2 k}(s, t) \sin k \theta\right], \\
& w(s, \theta, t)=-\sum_{k=1}^{\infty}\left[w_{1 k}(s, t) \sin k \theta-w_{2 k}(s, t) \cos k \theta\right] / k, \\
& u(s, \theta, t)=(\pi r / L) \sin (\pi s / L) \sum_{k=1}^{\infty}\left[w_{1 k} \cos k \theta+w_{2 k} \sin k \theta\right],
\end{aligned}
$$


A pipe bend obeys the hypothesis of flat sections. To derive the equations of motion, we use the second-order Lagrange Equations (Vlasov 1949):

$$
\frac{d}{d t}\left(\frac{\partial K}{\partial \dot{w}_{m n}}\right)+\frac{\partial \Delta}{\partial \dot{w}_{m n}}-\frac{\partial(K-\Pi-W)}{\partial w_{m n}}=Q_{m n}
$$

Here $Q_{m n}(t)$ is generalization of external forces related to generalized coordinates $w_{m n}(\mathrm{~m}=1,2$ and $n=1,2 \ldots$.$) .The motion of the time-averaged flow will be decomposed figurative and relative$ with velocity $\mathrm{V}_{\mathrm{m}}$. In this case, the kinetic energy of the unperturbed parts of the flow can be expressed as (Stasenko and Rakhmanova 1986)

$$
\begin{aligned}
& K=\frac{1}{2} \rho_{T} r \int_{-\frac{L}{2}}^{\frac{L}{2}} \int_{0}^{2 \pi}\left(\dot{u}^{2}+\dot{v}^{2}+\dot{w}^{2}\right) h(\theta) d s d \theta+\frac{1}{2} m_{\varkappa} \int_{-\frac{L}{2}}^{\frac{L}{2}}\left[\left(\dot{w}_{11}+V_{m} w_{21}^{\prime}\right)^{2}\right] d s= \\
& =\frac{1}{8}\left(m_{T}+m_{\varkappa}\right) L\left[\left(\frac{6 \pi-16}{\pi}+\frac{\pi^{2} r^{2}}{L^{2}}\right)\left(\dot{w}_{11}^{2}+\dot{w}_{21}^{2}\right)-e\left(\frac{12-3 \pi}{2 \pi}\right)\left(\dot{w}_{11}^{2} \dot{w}_{12}^{2}+\dot{w}_{21}^{2} \dot{w}_{22}^{2}\right)+\right. \\
& \left.+\sum_{n=2}^{\infty}\left(\frac{n^{2}+1}{n^{2}}+\frac{\pi^{2} r^{2}}{n^{4} L^{2}}\right)\left(\dot{w}_{1 n}^{2}+\dot{w}_{2 n}^{2}\right)-e \frac{n(n-1)+1}{n(n-1)}\left(\dot{w}_{1 n}^{2} \dot{w}_{1 n+1}^{2}+\dot{w}_{2 n}^{2} \dot{w}_{2 n+1}^{2}\right)\right]+m_{\varkappa} V_{m}^{2} \frac{\pi}{4 L}\left(\dot{w}_{11}^{2}+\dot{w}_{21}^{2}\right),
\end{aligned}
$$

where $m_{T} \approx 2 \pi \rho_{T} h_{m} r$ and $m_{\varkappa}=\rho_{\varkappa} A_{0}$ is pipe mass and fluid mass per unit length; $\rho_{T}$ and $\rho_{\varkappa}$ area average densities of composite and liquid.

The last term in the expression for $\mathrm{K}$ determines the potential of the centrifugal inertia forces of the fluid due to a change in the curvature of the axial line of the pipe. In view of the fact that the first harmonic is taken into account in the description of the rod forms (3), the Carioles inertia forces vanish. The viscoelastic potential, built on the basis of the relations of the semi - moment less theory of anisotropic layered shells and approximations (3), has the form:

$$
\begin{aligned}
& \Pi=\frac{1}{2} r \int_{-\frac{L}{2}}^{\frac{L}{2}} \int_{0}^{2 \pi}\left(B_{1} \varepsilon_{1}^{2}+D_{2} k_{2}^{2}\right) d s d \theta=\frac{\pi B_{1 m}}{4} r L\left\{\frac{\pi^{4} r^{2}}{L^{4}}\left(w_{11}^{2}+w_{21}^{2}\right)-\frac{\pi^{2} r}{L^{2}}\left[2\left(w_{11} g_{11}+w_{21} g_{21}\right)+\right.\right. \\
& \left.\left.+e\left(w_{11} g_{12}+w_{21} g_{22}\right)\right]+\sum_{n=1}^{\infty}\left[\left(g_{1 n}^{2}+g_{2 n}^{2}\right)-e\left(g_{1 n} g_{1 n+1}+g_{2 n} g_{2 n+1}\right)\right]\right\}+ \\
& +\frac{\pi D_{2 m} L}{4 r^{3}} \sum_{n=1}^{\infty}\left[\left(n^{2}-1\right)^{2}\left(w_{1 n}^{2}+w_{2 n}^{2}\right)-3 e n\left(n^{2}-1\right)(n+2)\left(w_{1 n} w_{1 n+1}+w_{2 n} w_{2 n+1}\right)\right] .
\end{aligned}
$$

Here $\tilde{E}_{1}, \tilde{E}_{2}$ are operator moduls of elasticity, which have the form

$$
E_{k} f(t)=E_{0 k}\left[f(t)-\int_{0}^{t} R_{E k}(t-\tau) f(\tau) d \tau\right], k=1,2
$$

where $f(t)$ is the time derivative function; $R_{E \kappa}(t-\tau)$ is core relaxation; $E_{0 \kappa}$ is instant modulus; $v_{12}, v_{12}$ are Poisson's ratios which are considered constant; 


$$
\begin{aligned}
& B_{1}(\theta) f(t)=(1-e \cos \theta) \tilde{B}_{1 m} f(t), \tilde{B}_{1 m} f(t)=\frac{h_{m}}{1-v_{12} v_{21}} \tilde{E}_{1} f(t) \\
& D_{2}(\theta) f(t) \approx(1-3 e \cos \theta) \tilde{D}_{2 m} f(t), \tilde{D}_{2 m} f(t)=\frac{h_{m}^{3}}{1-v_{12} v_{21}} \tilde{E}_{2} f(t) .
\end{aligned}
$$

The axial deformation of the shell has the form (Khaletskaya 1975)

$$
\begin{aligned}
& \varepsilon_{1}=\frac{\partial^{2} w_{11}(s)}{\partial s^{2}} r \cos \theta+\frac{\partial^{2} w_{21}(s)}{\partial s^{2}} r \sin \theta+\frac{1}{R}(w \cos \theta-v \sin \theta)= \\
& =\left[-\frac{\pi^{2} r}{L^{2}}\left(w_{11} \cos \theta+w_{21} \sin \theta\right)+\sum_{n=1}^{\infty}\left(g_{1 n} \cos n \theta+g_{2 n} \sin n \theta\right)\right] \cos \frac{\pi s}{L} \\
& g_{1 n}=\frac{n-2}{n-1} \frac{w_{1 n-1}}{2 R}+\frac{n+2}{n+1} \frac{w_{1 n+1}}{2 R}, \quad g_{2 n}=\frac{n-2}{n-1} \frac{w_{2 n-1}}{2 R}+\frac{n+2}{n+1} \frac{w_{2 n+1}}{2 R} .
\end{aligned}
$$

The change in the curvature of the wall in the circumferential direction has the following expression (Vasiliev 1988)

$$
k_{2}=\frac{1}{r^{2}}\left(\frac{\partial^{2} w}{\partial \theta^{2}}-\frac{\partial v}{\partial \theta}\right)=-\frac{1}{r^{2}} \cos \frac{\pi s}{L} \sum_{n=2}^{\infty}\left(n^{2}-1\right)\left(w_{1 n} \cos n \theta+w_{2 n} \sin n \theta\right) .
$$

The potential of external load moments $M_{m}$ and internal pressure $\left(p_{m}\right)$ in generalized coordinates has the form (Biderman 1980):

$$
W=-M_{m} \Delta \Phi-\hat{p}_{m} \Delta V=\frac{1}{2} \pi \hat{p}_{m} L\left\{\frac{2 \pi e r^{3}}{L^{2}} w_{11}-\frac{1}{2} \sum_{n=2}^{\infty}\left(n^{2}-1\right)\left[\left(w_{1 n}+\Delta_{n}\right)^{2}+w_{2 n}^{2}\right]\right\},
$$

where

$$
\begin{gathered}
\Delta V=r \int_{-\frac{L}{2}}^{\frac{L}{2}} \int_{0}^{2 \pi}\left[w-\frac{v}{2 r}\left(\frac{\partial w}{\partial \theta}-v\right)\right] d s d \theta=\frac{\pi}{4} L \sum_{n=2}^{\infty}\left(n^{2}-1\right)\left[\left(w_{1 n}+\Delta_{n}\right)^{2}+w_{2 n}^{2}\right], \\
\Delta \Phi=\int_{-\frac{L}{2}}^{\frac{L}{2}} \frac{\partial^{2} w_{11}(s, t)}{\partial s^{2}} d s=2 \frac{\pi}{L} w_{11} .
\end{gathered}
$$

Here $\Delta \Phi$ is mutual angle of rotation of the end sections due to bending of the pipe axis, and $\Delta V$ is the volume change due to Karman effect.

Using the Lagrange equations (2) and relations (3) - (5), we obtain two independent systems and integro - differential equations with variable stiffness coefficients of the form (Korotkov and Kulikov 2010):

$$
\begin{aligned}
& {[A]\{\ddot{w}\}+\alpha([C]-2 \mu(t)[F])\{w\}-\alpha[C] \int_{0}^{t}[R(t-\tau)]\{w(\tau)\} d \tau=-\bar{p}(t) \beta([F]\{\Delta\}+e\{P\}),} \\
& {\left[A^{\prime}\right]\{\ddot{w}\}+\alpha\left(\left[C^{\prime}\right]-2 \mu(t)[F]\right)\{w\}-\alpha\left[C^{\prime}\right] \int_{0}^{t}[R(t-\tau)]\{w(\tau)\} d \tau=0}
\end{aligned}
$$


Here $\{w\},\{\ddot{w}\}$ are vectors of generalized coordinates and accelerations,

$$
\alpha=\frac{6 \pi D_{2 m}}{m_{T} r^{3}}, \beta=\frac{2 \pi}{m_{T}}, \mu(t)=0.5 \bar{p}(t), \bar{p}(t)=\frac{r^{3}}{3 D_{2 m}} p(t)
$$

$D_{2 m}=\frac{h_{m}^{3}}{1-v_{12} v_{21}} E_{02},\{\Delta\}=\left\{0, \Delta_{1}, \Delta_{2}, \Delta_{3} \ldots \ldots \ldots,\right\}^{T}$ is the vector of amplitudes of initial shape irregularities; $e\{p\}=e\left\{\frac{2 \pi r^{3}}{L^{3}} 0,0 \ldots \ldots \ldots,\right\}^{T}-$ is the vector, describing the thickness of the pipe wall. Terms $|A|,|C|,|F|$ can be defined by the following formulas (Novozhilov et al. 1991):

$$
\begin{aligned}
& a_{11}=\left(\frac{6 \pi-16}{\pi}+\frac{\pi^{2} r^{2}}{L^{2}}\right)\left(\frac{m_{r}+m_{э}}{m_{r}}\right) ; \quad b_{11}=\frac{6 \pi-16}{\pi}+\frac{\pi^{2} r^{2}}{L^{2}} \\
& a_{1,2}=-\frac{3(4-\pi)}{4 \pi} ; \quad a_{n, n}=b_{n, n}=\frac{n^{2}+1}{n^{2}}+\frac{\pi^{2} r^{2}}{n^{4} L^{2}} ; a_{n, n+1}=-\frac{n(n+1)+1}{2 n(n+1)} e ; \\
& c_{11}=\frac{4}{\zeta \lambda^{2}} \eta^{2}-\tilde{\hat{N}}_{m} ; \quad c_{12}=-\frac{3}{\zeta \lambda^{2}} \eta ; \quad f_{n n}=n^{2}-1 \\
& c_{n, n}=\frac{4}{\zeta \lambda^{2}}\left[\delta_{n 2}\left(\frac{n+1}{n}\right)^{2}+\left(\frac{n-1}{n}\right)^{2}\right]+\frac{1}{3}\left(n^{2}-1\right)\left(n^{2}-1+3 \tilde{\hat{p}}_{m}\right) ; \\
& c_{n, n+1}=-\frac{e}{2 \zeta \lambda^{2}}\left[\frac{n+2}{n}+2 \frac{n-1}{n}+\lambda^{2} n\left(n^{2}-1\right)(n+2)\right] \text {; } \\
& c_{n, n+2}=\frac{1}{\zeta \lambda^{2}} \frac{(n-1)(n+3)}{n(n+2)} ; \quad c_{n, n+3}=-\frac{e}{\zeta \lambda^{2}} \frac{(n-1)(n+4)}{2 n(n+2)} ; \\
& \delta_{n 2}=\left\{\begin{array}{ll}
0 & ,
\end{array} \quad(n=2,3,4, \ldots) .\right.
\end{aligned}
$$

For the integral terms, the freezing method is applied (Ahmadi and Satter 1978), so that we obtain the following relations

$$
\zeta=\frac{E_{02}\left[1-\Gamma_{2}^{C}\left(\omega_{R}\right)-i \Gamma_{2}^{S}\left(\omega_{R}\right)\right]}{E_{01}\left[1-\Gamma_{1}^{C}\left(\omega_{R}\right)-i \Gamma_{1}^{S}\left(\omega_{R}\right)\right]}, \delta_{\eta}=\frac{\pi^{2} r R}{L^{2}}, \quad \lambda=\frac{R h}{r^{2}}, \quad \tilde{\hat{p}}_{m}=\frac{\hat{p}_{m} r^{3}}{3 D_{2 m}}, \quad \tilde{\hat{N}}_{m}=\frac{\pi r^{3}}{3 L^{2} D_{m}} \hat{N}_{m}
$$

$\Gamma_{k}^{C}\left(\omega_{R}\right)=\int_{0}^{\infty} R_{k}(\tau) \cos \omega_{R} \tau d \tau, \Gamma_{k}^{S}\left(\omega_{R}\right)=\int_{0}^{\infty} R_{k}(\tau) \sin \omega_{R} \tau d \tau$ - are dimensionless parameters; $\hat{N}_{m}=\hat{p}_{m} A_{0}-N_{m}$ is the longitudinal force ( $N_{m}$ meets initial stress - strain state). The matrix $|A|$ learns from three diagonal, the matrix $|C|$ is from three diagonal ribbon with complex coefficients and takes the following form. 
The matrix $|A|$ learns from the tridiagonal matrix, from the $|C|$ three-diagonal ribbon matrix with complex coefficients and assumes $\hat{N}_{m}=\hat{p}_{m}=0$ the following form:

$$
[C]=\left[\begin{array}{ccccc}
2 \delta_{\eta} & -1.5 \delta_{\eta} & 0 & 0 & 0 \\
-1.5 \delta_{\eta} & \delta_{22} & \delta_{23} & \frac{5}{16} & -\frac{3}{20} e \\
0 & \delta_{23} & \delta_{33} & \delta_{34} & \frac{2}{5} \\
0 & \frac{5}{16} & \delta_{34} & \delta_{44} & \delta_{45} \\
0 & -\frac{3}{20} e & \frac{2}{5} & \delta_{45} & \delta_{55}
\end{array}\right]
$$

Here

$$
\begin{gathered}
\delta_{22}=\frac{1}{8}+\frac{3}{2} \varsigma \lambda^{2}, \delta_{23}=-e\left(\frac{3}{4}+6 \lambda^{2}\right), \delta_{33}=\frac{10}{9}+\frac{32}{3} \varsigma \lambda^{2}, \delta_{34}=-e\left(\frac{3}{4}+30 \lambda^{2}\right) \\
\delta_{44}=\frac{17}{16}+\frac{225}{6} \varsigma \lambda^{2}, \delta_{45}=-e\left(\frac{3}{4}+90 \lambda^{2}\right), \delta_{55}=\frac{26}{25}+96 \varsigma \lambda^{2}
\end{gathered}
$$

The first equation (6) describes the forced parametric vibrations of the shell in the plane of its curvature. Oscillations are simultaneously supported by force and parametric excitation. Force excitation is initiated by initial geometric irregularities. The interaction of generalized $w_{1 n}(t)(n=1,2,3 \ldots)$ coordinates is determined by mutual inertial and viscoelastic bonds, the intensity of which is characterized by off-diagonal matrix elements $|A|$ and $|C|$. The second equation (5) describes the parametric vibrations of the shell (pipe) along the normal to the plane. Parametric excitation is due to changes in the shell stiffness associated with bending deformations of the shell. The matrix $|A|$ is obtained diagonal $(\mathrm{e}=0)$.

\section{Solutions of particular problems}

3.1. Consider the effect of harmonic pressure $p(t)=p_{k}(1+\chi \cos \Omega t)$, where $\chi=p_{0} / p_{k}$ - is the ripple parameter, $p_{0}$ and $\Omega$ are the amplitude and circular frequency. The mass of liquid is neglected. As an example, we consider the first two harmonics of a number of displacement functions (1).The interaction of generalized coordinates $w_{11}(t)$ and $w_{12}(t)$ neglected. In this case, the equations of motion (6) will take the following form:

$$
\begin{aligned}
& \ddot{w}_{11}(t)+\bar{\omega}_{1}^{2}\left(1-\Gamma_{n}^{\circ}\left(\omega_{R}\right)\right) w_{11}(t)+\frac{4 \pi^{3} r^{3} e}{(6 \pi-16) m_{p} L^{2}}\left(p_{m}+p_{0} \cos \Omega t\right)=0, \\
& \ddot{w}_{12}(t)+\bar{\omega}_{11}^{2}\left(1-\Gamma_{n}^{\circ}\left(\omega_{R}\right)-2 \mu \cos \Omega t\right) w_{12}(t)+\frac{24 \pi}{5 m_{p}}\left(p_{m}+p_{0} \cos \Omega t\right)=0,
\end{aligned}
$$

where 


$$
\begin{gathered}
\Gamma_{n}^{\circ}(\omega)=\Gamma_{k}^{C}(\omega)+i \Gamma_{k}^{S}(\omega), \omega_{1}^{2}=\frac{2 \pi^{5}}{(6 \pi-16) m_{p} L^{4}}\left(\frac{E_{01}}{1-v_{12} v_{21}}-\frac{\hat{N}_{m} L^{2}}{\pi^{2}}\right), \\
\omega_{11}^{2}=\frac{6 \pi}{5} \frac{1+12 \varsigma \lambda^{2}\left(1+\bar{p}_{k}\right) 2 \pi^{5}}{\varsigma \lambda^{2}} \frac{D_{2 m}}{m_{p} r^{3}}, \beta_{2}=\frac{24 \pi}{5 m_{p}}, \mu_{2}=\frac{6 \varsigma \lambda^{2} \bar{p}_{0}}{1+12 \varsigma \lambda^{2}\left(1+p_{m}\right)}, \\
\bar{p}_{k}=\frac{p_{k} r^{3}}{3 D_{2 k}}, \bar{p}_{0}=\frac{p_{0} r^{3}}{3 D_{2 k}}, I_{z} \approx \pi r^{3} h_{m}
\end{gathered}
$$

Here $\omega_{1}$ and $\omega_{11}$ are the partial complex frequencies.

The first equation (7) describes the forced vibrations (cantilever) of a viscoelastic rod of length $\mathrm{L} / 2$ under the action of a focusing moment. If we accept $v_{12}(t)=R_{K}=0$, then the error in the natural frequency of transverse vibrations $\omega_{1}=\omega_{I}$ will be $4.2 \%$ [20].

The second equation (7) describes the forced parametric vibrations of the shell under the action of pressure $\mathrm{p}(\mathrm{t})$. In the case $\frac{1}{R} \rightarrow 0$, the expression for coincides with the formula for $\omega_{2}=\omega_{I I}$ the fundamental frequency of the bending vibrations of the circular shell (Timoshenko et al. 1985).

3.2. In the absence of pulsations ( $p_{0}=0, p_{m} \neq 0$ ), the solution of the quasistatic problem follows from the first equation (6):

$$
[C]\left(\{w\}-\int_{0}^{t}[R(t-\tau)]\{w(\tau)\} d \tau\right)=-p_{k}([F]\{\Delta\}+e\{P\})
$$

Solution (8) determines the generalized displacements of a curved composite pipe with initial ovality and thickness variation under the influence of static pressure $\mathrm{p}_{\mathrm{m}}$. With an absolutely rigid contour of the cross section of the pipe, $R(t-\tau)=0$ we get a beam. In this case, from equation (8) we find (Korotkov and Kulinov 2010)

$$
w_{11}=-\frac{2}{\pi^{2}} p_{k}\left(1-v_{12} v_{21}\right) e r^{3} L^{2} /\left(E_{01} I_{z}\right)
$$

Expression (9) determines the displacement of the free end of a curved composite pipe in pure bending.

3.3. In the absence of initial irregularities and the action of harmonic pressure, the first integrodifferential equation (6) becomes homogeneous and coincides with the second equation:

$$
\left[A^{\prime}\right]\{\ddot{w}\}+\alpha\left(\left[C^{\prime}\right]-2 \mu(t)[F] \cos \Omega t\right)\{w\}-\alpha\left[C^{\prime}\right] \int_{0}^{t}[R(t-\tau)]\{w(\tau)\} d \tau=0 .
$$

When $\frac{1}{R} \rightarrow 0$, the matrix $\left[C^{\prime}\right]$ is reduced to diagonal form. In this case, the coupled system of integro - differential equations (10) decomposes to a system of independent Mathieu equations with a complex output parameter is separated from it

$$
\ddot{w}_{n}+\bar{\omega}_{n}^{2}\left(1-\Gamma_{n}^{\circ}\left(\omega_{R}\right)-2 \mu_{n} \cos \Omega t\right) w_{n}=0(n=2,3,4 \ldots),
$$

where 


$$
\omega_{m n}^{2}=\frac{2 \pi D_{2 m} n^{2}\left(n^{2}-1\right)\left(n^{2}-1+3 \tilde{p}_{m}\right)}{m_{T} r^{3}\left(n^{2}+1\right)}, \mu=\frac{3 \tilde{p}_{0}}{2\left(n^{2}-1+3 \tilde{p}_{m}\right)}, \Gamma_{n}^{\circ}(\varpi)=\Gamma_{k}^{C}(\varpi)+i \Gamma_{k}^{S}(\varpi)
$$

Equations (11) describe the parametric oscillations of a cylindrical shell and coincides with the known solutions for $R(t-\tau)=0 \quad$ [20]. When $\omega_{n}=0$ we get:

$$
p_{\text {crit }}=p_{m}=\left(n^{2}-1\right) D_{2} / r^{2}
$$

Formula (12) determines the spectrum of critical values of the external pressure at which the cylindrical shell loses its static stability (Alfutov 1978).

The resolving equation (11) with $R(t-\tau)=0$, in the general case, a system of separable equations is reduced $(\mathrm{m}, \mathrm{n}=1,2,3 \ldots)$ quation of Mathieu:

$$
\ddot{w}_{n}(t)+\bar{\omega}_{m n}^{2}\left(1-\delta_{m n} \cos \Omega t\right) w_{n}(t)=0
$$

where $\delta_{m n}$ - is the excitation coefficient, defined by the expression:

$$
\delta_{m n}=\frac{m^{4}\left(m^{2}-1\right) q_{0}^{*}}{\left[\lambda_{n}^{4}+m^{4} \eta\left(m^{2}-1\right)\left(m^{2}-1-\frac{q_{0}^{*}}{\eta}\right)+k^{*} \lambda_{n}^{2} m^{4}\right]} \cdot \mu
$$

where

$$
q_{0}^{*}=G q_{0}, G=R_{0} / E_{0} h h_{v}^{2}
$$

The solution of the Mathieu differential equation (13) allows one to construct regions of dynamic instability of structures.

Assessment of the dynamic stability of pipelines lying on an elastic foundation consists, firstly, in constructing regions of dynamic instability on the parameter plane $\omega_{\mathrm{mn}}$ and $\Omega$ under a given condition of external pressure $\mathrm{q}_{0}$ and various values of the soil bed coefficient K. Secondly, a direct assessment of the dynamic stability of a given section of the pipeline is carried out at known values of $\omega_{\mathrm{mn}}, \Omega$ and $\mathrm{q}_{0}$ by superimposing a point corresponding to these values on the parameter plane $\Omega, \mathrm{q}_{0}$, containing the region of dynamic instability (Safarov et al. 2018).

The regions of dynamic instability are determined at the frequency ratios $\omega_{\mathrm{mn}}$ and $\Omega$. The main, widest region, called the main region of instability, is realized for the coefficients $\mathrm{k}=1$, i.e., for $\omega_{m n}=\Omega / 2$. The minor instability regions for $\mathrm{k}>1$ are much smaller in width and usually overlap with the main region. The solution of the Mathieu equation in notation (13) for the main instability region obtained in the work of N.N. Bogolyubov and Yu.A. Metropolitan, represents the inequality (Safarov et al. 2018):

$$
1-\frac{\delta_{m n}}{2}<\left[\frac{2 \omega_{m n}}{\Omega}\right]<1+\frac{\delta_{m n}}{2}
$$

Based on this solution, the methodology for constructing the main areas of dynamic instability for pipeline sections is to determine the upper and lower boundaries of these areas. 


\section{Numerical results}

The calculation results are shown in Figs. 1-2. The methodology for assessing the dynamic stability of the pipeline is reduced to finding the position of the point $\left(\Omega, q_{0}\right)$.

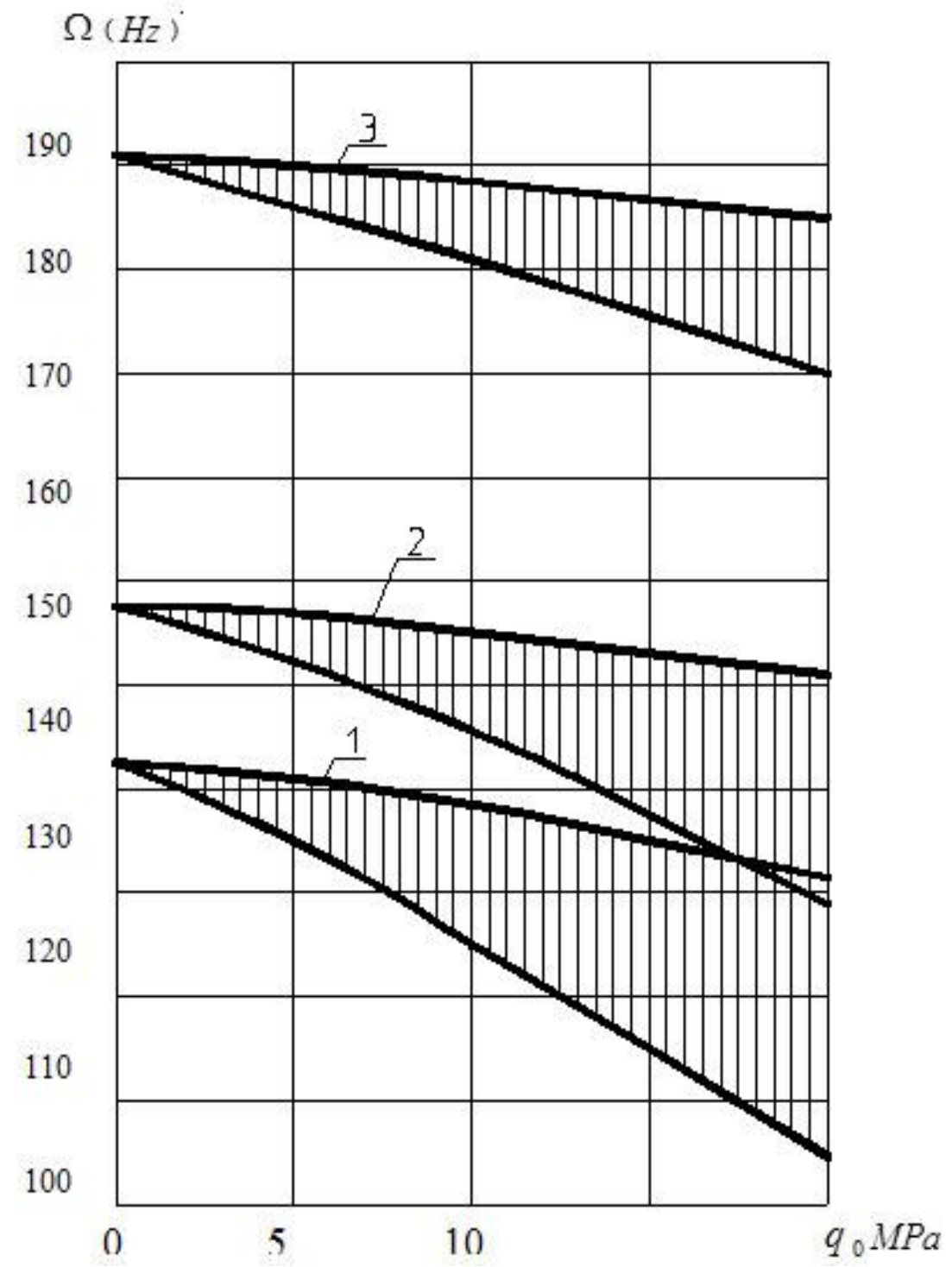

Fig. 1. Areas of dynamic instability of a metal pipeline $(1420 \times 10 \mathrm{~mm})$ with external pressures at different thicknesses of the protective reinforced concrete layer $1-\mathrm{h}_{1}=45 \mathrm{~mm}, 2-\mathrm{h}_{1}=65$ $\mathrm{mm}, 3-\mathrm{h}_{1}=85 \mathrm{~mm}$. 


\section{$\Omega(\mathrm{Hz})$}

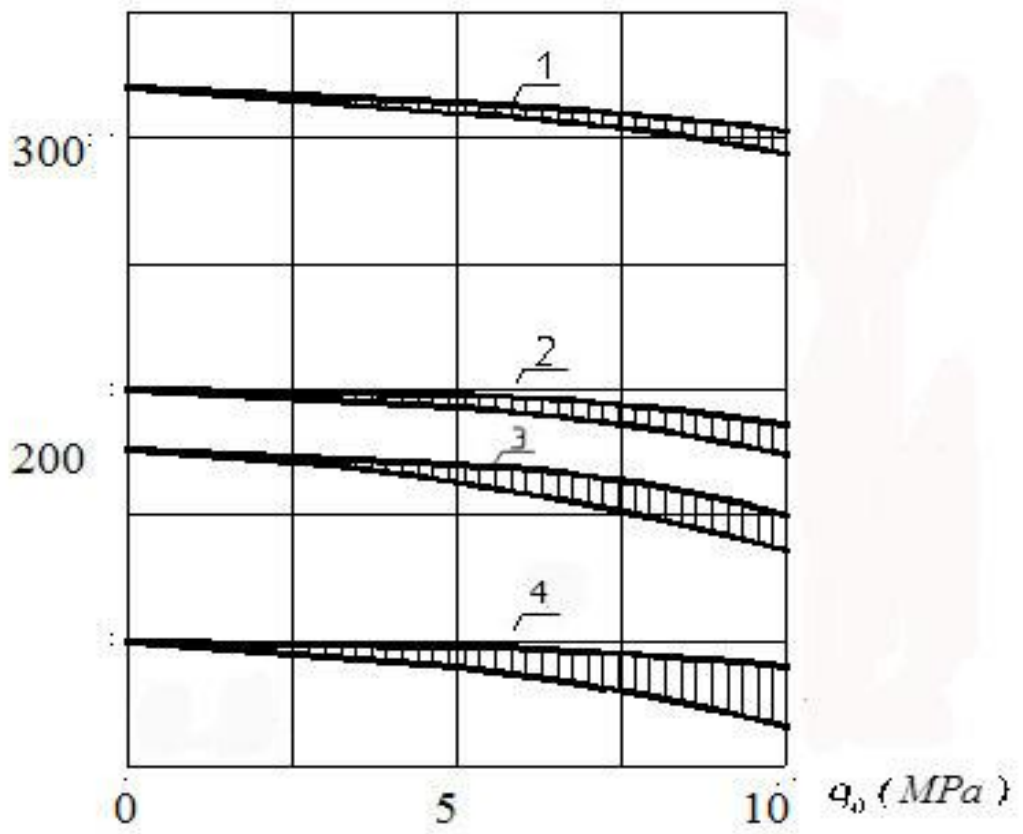

Fig. 2. Areas of dynamic instability of curvilinear pipelines at $r / R=1 / 10$ and with different thin-walled parameters under $h / r(1 . h / r=1 / 12 ; 2 . h / r=1 / 15 ; 3 . h / r=2 / 35 ; 4 . h / r=1 / 20$. $)$ the influence of external unsteady pressure $\mathrm{q}_{0}$.

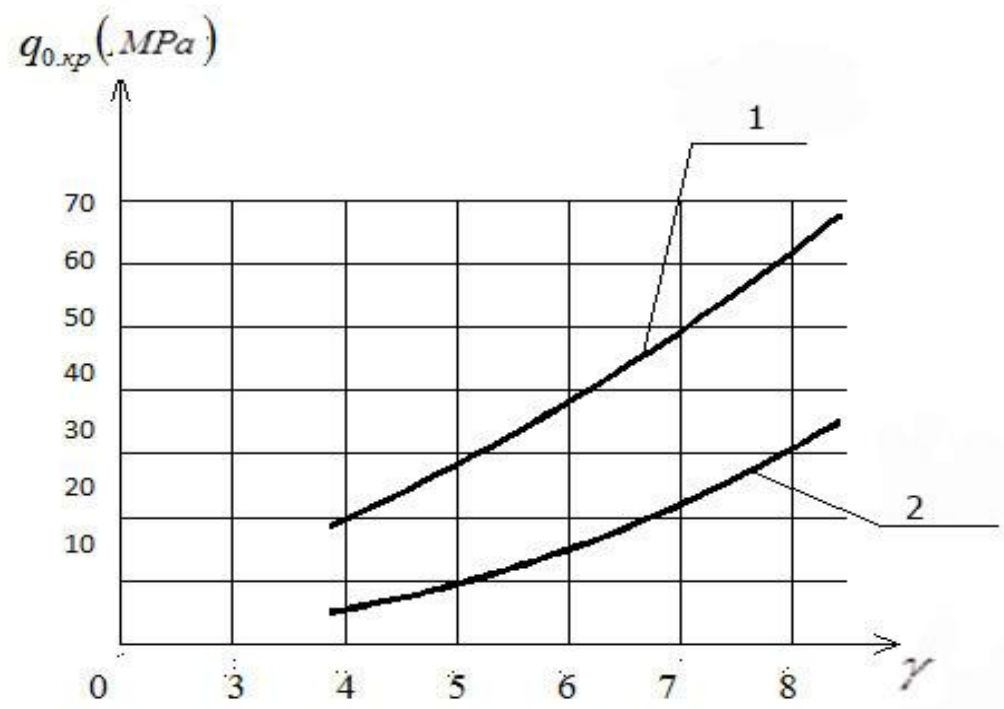

Fig. 3. Dependence of the critical external pressure $\mathrm{q}_{0, \mathrm{cr}}$ on the thickness parameter and relative curvature. $1 \cdot \frac{r}{R}=0.1 ; 2 \cdot \frac{r}{R}=\frac{1}{30}$. 
If this point falls on a plane free of shaded instability regions, this pipeline stability is ensured. Otherwise, you should change the main parameters of the pipeline $\left(\omega_{\mathrm{mn}}, \mathrm{q}_{0}\right.$ or $\left.\Omega\right)$. Firstly, the oscillation frequencies decrease with a decrease in the $\mathrm{h} / \mathrm{r}$ parameter with a fixed value of the pipe curvature parameter $r / R$. The rate of frequency reduction is higher, the smaller the parameter $\mathrm{h} / \mathrm{r}$. Secondly, with a decrease in the curvature of the pipeline section, that is, the $r / R$ ratio at $h / r=$ const, the frequency (Fig. 1 and 2).The calculations showed $q_{0, c r}$ that it substantially depends on the parameters $\mathrm{h} / \mathrm{r}$ and $\mathrm{r} / \mathrm{R}$. This dependence is illustrated by the graphs in Fig. $3\left(\gamma=\frac{h}{R} 10^{2}\right)$. Analysis of the obtained values shows that the critical external pressure increases with increasing thickness parameter $\mathrm{h} / \mathrm{r}$. It should be noted that for pipes with large curvature $\mathrm{r} / \mathrm{R}=1 / 10$, the critical pressure value is greater than for pipes with lesser curvature $r / R=1 / 30$.

\section{Conclusions:}

Analysis of the calculation results at an external pressure acting on the pipe wall showed the following:

1. The lowest frequency of free bending vibrations of pipeline sections is realized by shell forms, that is, $\omega_{21}$ and $\omega_{31}$ for $\mathrm{m}=2,3$ and $\mathrm{n}=1$.

2. With an increase in the pipe curvature parameter, $\mu$ the frequencies of free bending vibrations of the pipeline sections $\omega_{\mathrm{mn}}$ for $\mathrm{m}=1,2,3$ and $\mathrm{n}=1$ increase significantly.

3. When $\mathrm{h} / \mathrm{R}$ decreases with constant pipe curvature, a decrease in frequencies occurs.

\section{References}

Ahmadi G, Satter Mi (1978). Stability of a pipe carried time-dependent flowing! Fluids // J. Franklin Inst.305, 1, Pil-9.

Alfutov NA (1978). Basics of calculating the stability of elastic systems. Moscow, Mechanical engineering.

Amabili M, Pellicano F, Vakakis AF (2000). Nonlinear vibrations and multiple resonances of fluid-filled circularcylindrical shells. Part 1: Equations of motion and numerical results. Journal of Vibration and Acoustics. 122, 346-354

Biderman VL (1980). Theory of mechanical vibrations. Moscow, Higher School.

Hambartsumyan SA (1974). The general theory of anisotropic shells. -Moscow, Nauka press.

Khaletskaya OB (1975). Free vibrations of a thin-walled curved pipe. News of universities. Construction and architecture. 4, 34-39.

Korotkov AV, Kulikov YuA (2010). Free vibrations of multilayer composite curved pipes with flanges. Bulletin of KSTU named after A.N. Tupolev. 1 (57), 69-74.

Korotkov AV, Kulikov YuA (2010). Vibration resistance of multilayer curved pipes made of reinforced plastics. Mechanics of composite materials and construction. 16, 4, 178-194.

Kovalchuk PS, Kruk LA (2000). On nonlinear energy exchange between the intrinsic forms of circular cylindrical shells filled with liquid during their free vibrations // Prikladnaya mechanika, 36, 1,115-122

Kubenko VD, Kovalchuk PS, Boyarshina LG et al. (1992). Nonlinear dynamics of axisymmetric bodies carrying a fluid. Kiev, Ukraine, Naukova Dumka

Kubenko VD, Kovalchuk PS, Kruk L.A. (2003) Non-linear interaction of bending deformations of free oscillating cylindrical shells. Journal of Sound and Vibration, 265, 245-268 
Novozhilov VV, Chernykh KF, Mikhailovsky EI (1991). Linear theory of thin shells. Leningrd, Polytechnic.

Safarov II, Teshaev M Kh, Boltaev ZI, Akhmedov M Sh (2018a). Mathematical modeling of dynamic processes in a toroidal and cylindrical shell interacting with a liquid. Raleigh, North Carolina, USA: Open Science Publishing.

Safarov II, Teshaev MKh, Akhmedov MS (2018b). Free Oscillations of a Toroidal Viscoelastic Shell with a Flowing Liquid. American Journal of Mechanics and Applications. 6(2), 37-49

Safarov II, Boltaev ZI(2018). Methods for Assessing the Seismic Resistance of Subterranean Hydro Structures Under the Influence of Seismic Waves. American Journal of Physics and Applications, 6(2), 51-62. doi:10.11648/j.ajpa.20180602.14.

Safarov II, Teshaev M Kh, Boltayev Z I, Nuriddinov BZ (2018c). Own Waves in a Cylindrical Shell in Contact with a Viscous Liquid. Global Journal of Science FrontierResearch: Mathematics and Decision Sciences. 18, 1, 65-84

Safarov I.I., Teshaev M. Kh., Boltaev Z.I., Akhmedov M.Sh. (2018) Mathematical modeling of dynamic processes in a toroidal andcylindrical shell interacting with a liquid. Raleigh, NorthCarolina, USA: Open Science Publishing.

Safarov II, Teshayev MKh, Boltayev ZI, Nuriddinov BZ (2018). Own Waves in a Cylindrical Shell in Contact with a Viscous Liquid. World Wide Journal of Multidisciplinary Research and Development (WWJMRD), 4(1), 140-151.

Stasenko IV, Rakhmanova II (1986). Calculation of thin-walled pipes of large curvature. Strength calculations. Moscow, Mechanical Engineering, 27, 57-69.

Vasiliev VV (1988). Mechanics of structures made of composite materials. Moscow, Engineering.

Vibration in technology: Handbook. In 6 volumes. Volume 1. Oscillations of linear systems. (1978) Moscow, Mechanical Engineering.

Vlasov VZ (1949). The general theory of shells and its application to technology. Moskow, Gostekhizdat.

Timoshenko SP, Young DH, Weaver W. (1985). Fluctuations in Engineering. Moscow, Engineering. 